Projets

de paysage

\section{Projets de paysage}

Revue scientifique sur la conception et l'aménagement de l'espace

$8 \mid 2012$

Les concepteurs de jardins et de parcs japonais

\title{
Les atlas des paysages de Wallonie
}

L'exemple d'une approche multidisciplinaire du territoire intégrant un volet sociologique

The Atlases of Wallonia's Landscapes - The Example of a Multidisciplinary

Approach of the Territory, including a Sociological Constituent

Thaïs Pons, Marie-Françoise Godart et Mireille Deconinck

\section{OpenEdition}

\section{Journals}

Édition électronique

URL : http://journals.openedition.org/paysage/15228

DOI : $10.4000 /$ paysage. 15228

ISSN : 1969-6124

Éditeur :

École nationale supérieure du paysage de Versailles-Marseille, Institut national des sciences appliquées Centre Val de Loire - École de la nature et du paysage, École nationale supérieure d'architecture et de paysage de Bordeaux, École nationale supérieure d'architecture et de paysage de Lille, Agrocampus Angers

\section{Référence électronique}

Thaïs Pons, Marie-Françoise Godart et Mireille Deconinck, "Les atlas des paysages de Wallonie », Projets de paysage [En ligne], 8| 2012, mis en ligne le 13 juillet 2012, consulté le 11 mars 2021. URL http://journals.openedition.org/paysage/15228 ; DOI : https://doi.org/10.4000/paysage.15228

Ce document a été généré automatiquement le 11 mars 2021.

Projets de paysage 


\section{Les atlas des paysages de Wallonie}

L'exemple d'une approche multidisciplinaire du territoire intégrant un volet sociologique

The Atlases of Wallonia's Landscapes - The Example of a Multidisciplinary

Approach of the Territory, including a Sociological Constituent

Thaïs Pons, Marie-Françoise Godart et Mireille Deconinck

\section{NOTE DE L'AUTEUR}

Les atlas des paysages de Wallonie sont produits par la Conférence permanente du développement territorial(CPDT). L'objet de cet article consiste à faire connaître les atlas et en particulier les résultats de l'approche sociologique. Une bibliographie plus complète peut être consultée dans les atlas en ligne sur le site de la CPDT (http:// cpdt.wallonie.be).

\section{Contexte de l'étude}

1 Face à l'évolution rapide des paysages dans les pays européens et la perte d'identité culturelle et territoriale des habitants engendrée par cette évolution, le Conseil de l'Europe a réagi et s'est engagé à susciter la prise en compte du paysage dans diverses politiques. L'adoption de la Convention européenne du paysage à Florence en 2000 est à cet égard une étape cruciale. Celle-ci a pour objet de promouvoir la protection, la gestion et l'aménagement des paysages européens, que ceux-ci soient remarquables, ordinaires ou dégradés, naturels, urbains, périurbains ou ruraux. Le paysage est reconnu au sein de cette Convention comme une composante essentielle de la qualité de vie et comme facteur d'attractivité sociale et économique des territoires. Chaque partie signataire de la Convention s'engage à «identifier ses propres paysages sur l'ensemble de son territoire, à analyser leurs caractéristiques ainsi que les dynamiques et les pressions qui les modifient; à en suivre les transformations; à qualifier les 
paysages identifiés en tenant compte des valeurs particulières qui leur sont attribuées par les acteurs et les populations concernées ». CEP, Art.6c, Florence 2000.

2 Dans cette optique, la Wallonie (région du sud de la Belgique) s'est engagée à réaliser des travaux d'identification et de qualification de ses paysages.

\section{Méthodologie générale et objectifs des atlas}

3 Le travail entamé il y a 5 ans a débuté par une première étape menée à l'échelle du $1 / 50000^{\mathrm{e}}$ qui a consisté à identifier les différents paysages de la Wallonie et à les regrouper en 13 ensembles paysagers (carte 1a). La deuxième étape, toujours en cours, menée cette fois à l'échelle du $1 / 20000^{\mathrm{e}}$, vise à examiner ces ensembles plus en détail en vue de réaliser un atlas des paysages pour chacun d'eux et d'obtenir à terme une collection qui couvrira tout le territoire. À cette étape, les ensembles paysagers sont décomposés en "aires paysagères ", des portions de l'espace qui se différencient des espaces adjacents par les paysages qu'ils présentent (carte $1 b$ ).

Carte 1. Découpage de la Wallonie en ensembles paysagers et découpage de ces derniers en aires paysagères pour l'ensemble du plateau condrusien

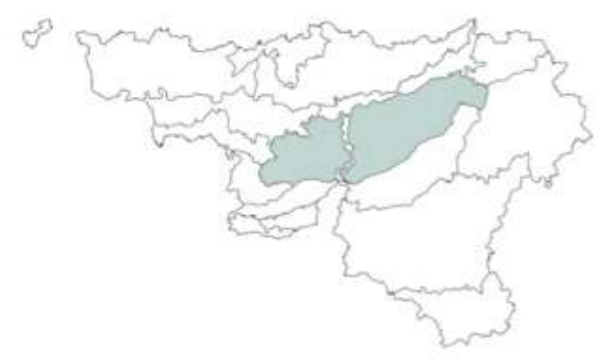

a) La Wallonie est subdivisée en 13 ensembles paysagers.

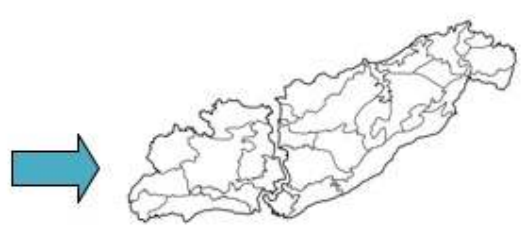

b) Chaque ensemble paysager est découpé en aires paysagères.

4 L'étude est encadrée par un groupe d'experts composé de personnes de référence dans le milieu académique, politique et de l'aménagement du territoire régional. À ce jour quatre atlas ont été réalisés, dont trois pour des ensembles à dominante rurale et un plus urbain.

5 L'objectif des atlas est de transmettre, à travers un socle commun, une analyse rigoureuse et générale de l'aménagement des différents espaces qui composent la Wallonie. Ces ouvrages apportent une contribution significative à la compréhension des patrimoines paysagers, à leur appréciation par le public et in fine à leur préservation. Alliant rigueur scientifique et vulgarisation de qualité, ils constituent un outil de sensibilisation, de gestion et de communication qui s'adresse à un large public, tant aux aménageurs et responsables politiques locaux qu'aux enseignants, associations d'habitants et à tout particulier sensible au patrimoine et à la connaissance de son cadre de vie. La collection des atlas se veut être un document de référence et d'orientation sans valeur réglementaire mais visant à susciter ou à permettre une harmonisation des décisions d'aménagement. 


\section{Quatre approches du paysage}

6 Plus qu'une analyse purement descriptive, il s'agit d'une étude interdisciplinaire combinant les sciences géographique, historique, écologique et sociologique. Ces disciplines posent un regard croisé tant au niveau global que détaillé. La méthode adoptée procède par quatre grandes approches visant une étude temporelle et spatiale élargie. Les trois premières sont ici résumées tandis que la quatrième, qui concerne l'aspect sociologique, fait l'objet d'une explicitation plus exhaustive de la méthode et des résultats obtenus.

\section{L'identification des caractéristiques visuelles}

7 Le relief, dans ses formes principales et secondaires, son altitude et son modelé, constitue le premier niveau d'analyse. Il s'agit d'un élément structurant des paysages dans la mesure où il influence la nature et la perception de l'espace. À un deuxième niveau, les interactions entre le substrat, la nature et les activités humaines ont été prises en compte à travers l'analyse de l'occupation du sol. Celle-ci se caractérise par la présence d'espaces bâtis et non bâtis, par leur répartition par rapport au relief (sitologie) ainsi que par les dynamiques qui les affectent. Pour les espaces non bâtis, la structure et la composition des espaces agricoles, forestiers et naturels, ainsi que leur mode d'agencement sont examinés. Les espaces bâtis sont analysés sous l'angle du bâti traditionnel (structure ancienne et typologie de l'habitat, présence de l'industrie) et de l'urbanisation récente. Les délimitations établies sur ces deux niveaux reflètent les limites de perception visuelle des unités paysagères composant un territoire.

\section{L'histoire des paysages}

8 L'étude de l'évolution historique des paysages couverts par l'atlas a pour but d'éclairer les caractéristiques actuelles des paysages. Pour ce faire, un travail de documentation permet de retracer les facteurs d'évolution des paysages sous l'effet des pratiques des sociétés humaines. Les aspects " hérités ", et toujours visibles, des paysages sont mis en exergue afin de comprendre et de mettre en évidence les mécanismes évolutifs de l'espace. Les pratiques agricoles, commerciales et culturelles, de même que l'organisation des sociétés et leur évolution politique, sont mises en relation avec les structures territoriales visibles. Ce volet présente une clé de lecture essentielle des paysages et de leurs caractéristiques actuelles, héritage d'actions et de modes de vie passés. Il permet de livrer aux acteurs et aux utilisateurs des atlas une objectivation des facteurs de mise en place des paysages actuels.

\section{La mise en lumière des évolutions et des enjeux}

9 Tant à l'échelle des aires, qu'à celle plus générale de l'ensemble, des enjeux sont identifiés et permettent la mise en avant des pressions, des évolutions probables ou des potentialités de développement en se ralliant aux concepts de gestion/aménagement/ protection des paysages préconisés par la Convention de Florence. Il s'agit de pistes de réflexion destinées à être débattues de manière plus approfondie entre acteurs du territoire, tant locaux que régionaux. Il a été volontairement décidé de proposer des 
pistes d'action pour chaque aire paysagère plutôt que des outils ou règlements précis à utiliser, ceux-ci étant trop rapidement obsolètes.

Une présentation synthétique sous forme de carte (carte 2) des grandes caractéristiques paysagères et des pressions majeures offre une vision globale à l'échelle de l'ensemble et rappelle l'importance d'adopter une réflexion qui dépasse les frontières administratives.

Carte 2. Caractéristiques et tendances des plateaux brabançon et hesbignon

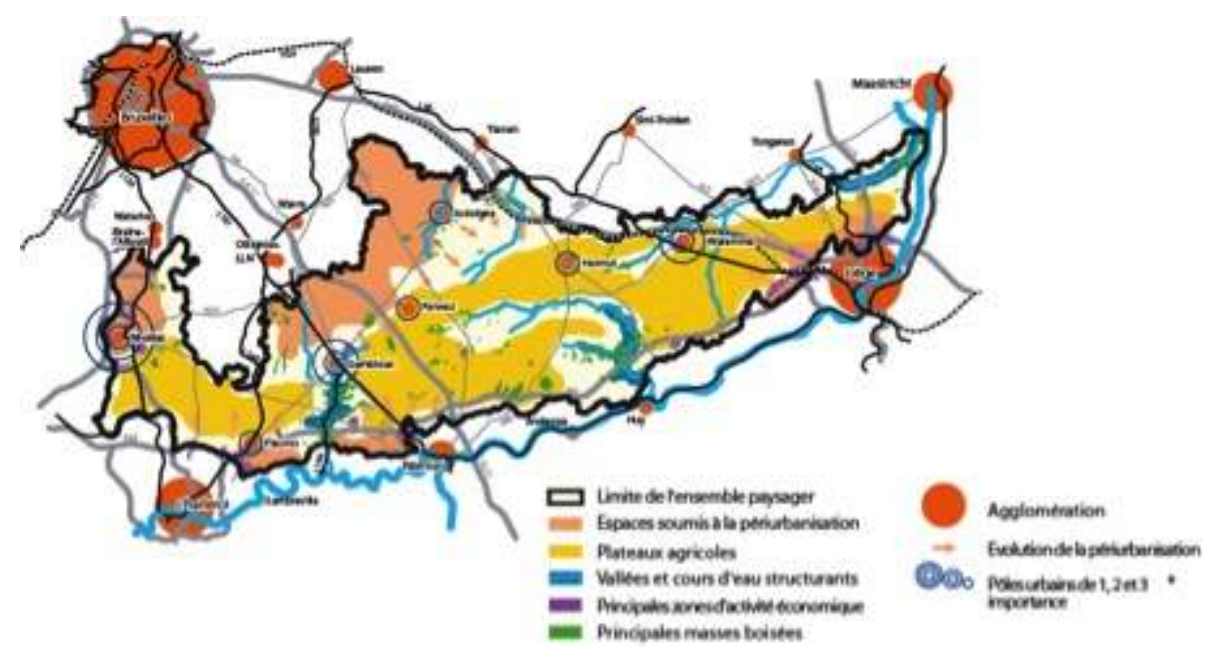

La pression sur les campagnes qui émane des grands pôles urbains comme Liège ou Namur au sud de l'ensemble est ici illustrée. Dans l'ouvrage, cette carte schématique est accompagnée d'une légende qui développe chacune des informations.

\section{L'approche sociologique : l'étude des perceptions des paysages}

La multiplication des études paysagères depuis ces dernières années ne doit pas faire oublier la difficulté qu'engendre la compréhension de la notion de paysage. Il importe de rappeler que le paysage n'existe qu'à travers l'interprétation qui en est faite à partir d'une combinaison d'éléments objectifs et de leur interprétation subjective. C'est dans ce sens qu'Augustin Berque (1994) considère que les matériaux qui constituent les paysages "n'acquièrent d'existence paysagère qu'à partir du moment où une symbolique collective en fait l'emblème d'une certaine identité. »

La Convention de Florence reconnait la dimension identitaire des paysages et stipule que les États signataires s'engagent «[...] à mettre en place des procédures de participation du public, des autorités locales et régionales, et des autres acteurs concernés par la conception et la réalisation des politiques du paysage » (art. 5c) et de «formuler des objectifs de qualité paysagère pour les paysages identifiés et qualifiés, après consultation du public. » (art. $6 \mathrm{~d})$.

Une partie sociologique s'est ainsi imposée lors de l'élaboration des atlas des paysages comme approche indispensable visant à cerner les référents imaginaires qui composent le milieu perçu par les individus, ainsi que la conscience d'appartenance des individus à un lieu de vie particulier. Ce regard social complète l'approche dite objective menée par la démarche scientifique dans les autres parties de l'ouvrage. L'appréhension du regard social passe ici par une démarche qualitative. 

communaux, de représentants d'associations liées au paysage, de représentants régionaux et d'acteurs en relation avec le monde agricole ont permis d'obtenir un matériau d'analyse qui couvre une bonne partie des quatre territoires étudiés. les plus intéressantes au niveau paysager, les endroits les plus typiques ou encore ceux qu'ils estiment abîmés. La discussion aborde ensuite les menaces, les enjeux, le rôle des acteurs et leurs actions en faveur du paysage. Certaines rencontres sont en outre complétées par une visite de terrain, permettant d'enrichir les propos tenus ainsi que de vivre et de partager les paysages identifiés sur la carte. Pour mieux cerner les perceptions des acteurs, il s'agit également de comprendre les raisons de l'attachement ou du rejet. Il en ressort un regard approfondi sur les objets et les thèmes paysagers auxquels les individus sont attachés.

Une carte intitulée « Paysages choisis " par les acteurs est présentée dans chaque atlas (carte 3). Elle reprend les sites ou points de vue les plus appréciés ou abîmés selon l'opinion des personnes interrogées. Les paysages médaillés sont ceux qui ont une résonance particulière parmi l'ensemble des paysages sélectionnés. Loin d'être une démarche exhaustive, cette sélection de paysages appréciés a l'avantage de mettre l'accent sur les caractéristiques qui laissent un souvenir spécifique auprès des acteurs locaux. Les paysages emblématiques qui ont une reconnaissance sociétale via un classement ne sont pas les seuls à marquer les esprits, des lieux plus intimistes peuvent aussi laisser un souvenir particulier pour les acteurs.

Carte 3. Les paysages choisis par les acteurs interrogés

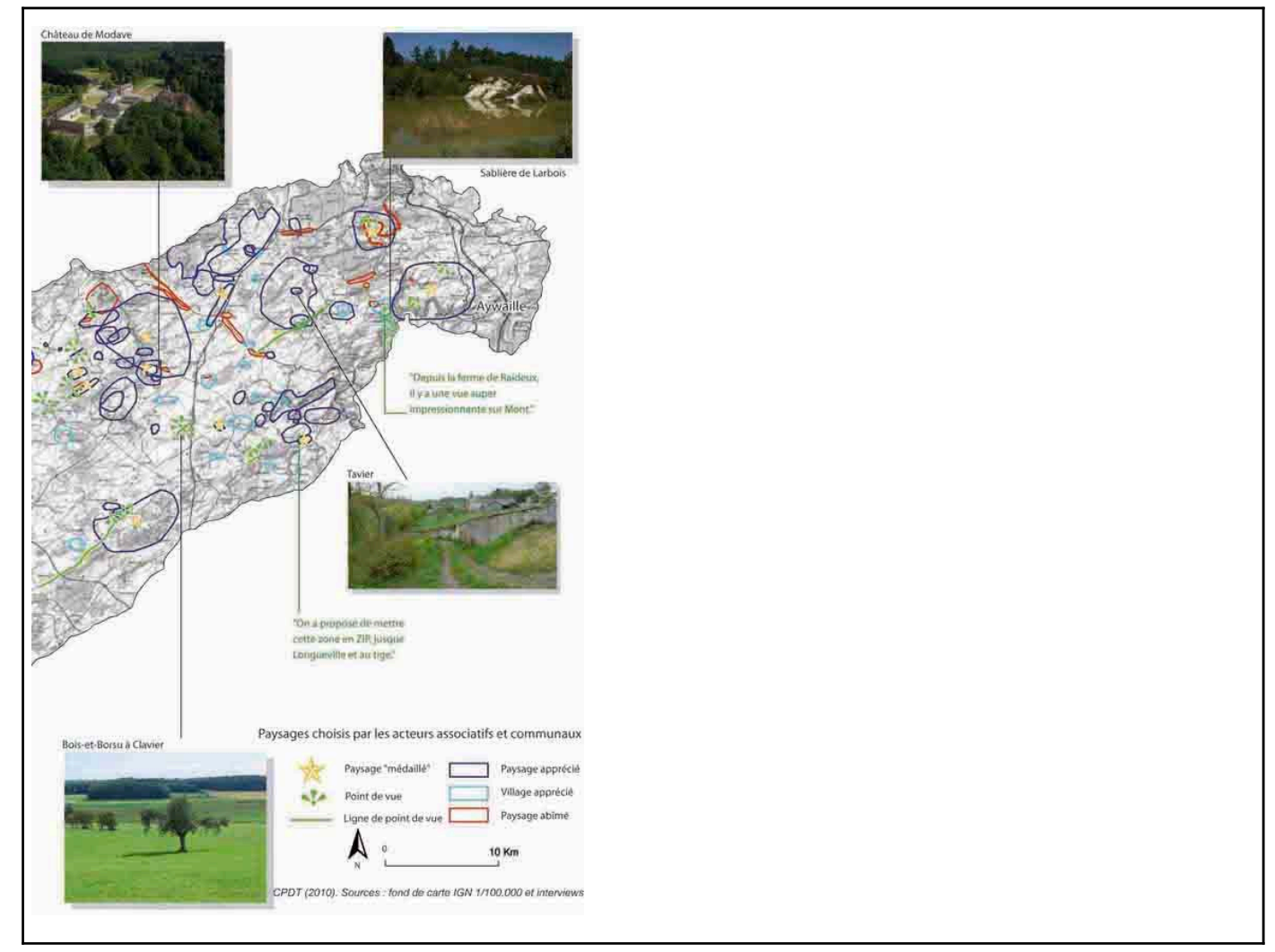


La carte 3 est un extrait de carte des «Paysages choisis » par les acteurs, présentée dans l'atlas des paysages du plateau condrusien. Elle montre une grande majorité de points de vue identifiés à proximité du relief offert par les vallées de la Meuse et de l'Ourthe, ainsi qu'au niveau de la bordure famennienne. Ces localisations de promontoires sont parfois préférées pour éviter le tracé d'un périmètre précis. Remarquons aussi qu'un lieu peut être sélectionné dans sa version restreinte ou plus étendue selon les personnes interrogées.

Pour les paysages à dominante rurale, à travers la diversité des lieux choisis, il apparaitt que la construction du discours sur les lieux les plus appréciés renvoie à trois schèmes de pensée (figure 1) et à cinq qualités essentielles (figure 2). Ceux-ci révèlent avec force le postulat posé par Augustin Berque (1994) selon lequel « les sociétés interprètent leur environnement en fonction de l'aménagement qu'elles en font et réciproquement, elles l'aménagent en fonction de l'interprétation qu'elles en font ».

18 Ainsi, les trois schèmes de pensée (figure 1), qui ont été identifiés au fil des interviews sont interconnectés et constituent autant d'entrées possibles vers les paysages et leur appréciation. L'image mentale d'un paysage passe par la sélection d'« objets paysagers » signifiants comme la présence d'eau, de bocages et de vergers, d'anciennes fermes, etc. : « Ce qui est sympathique dans les paysages de la Hesbaye, c'est si on peut retrouver un peu de nostalgie, une grosse ferme en carré, avec un chemin en pavés». Ces éléments du paysage renvoient à des thèmes plus généraux en lien avec la structure du paysage comme la nature, la diversité, l'accessibilité au paysage. Ces objets et thèmes essentiels peuvent être regroupés en catégories dont la synthèse des pressions en restitue la meilleure expression. Ainsi, faire référence à l'impact paysager de la disparition progressive des chemins creux ou de zones humides est la traduction d'une forte pression sur la biodiversité. Ou encore, souligner l'attachement particulier à un village "ancien» dont est appréciée la structure homogène, et rejeter systématiquement des nouvelles constructions de type "clé sur porte " le long des voies d'accès sont l'occasion de s'attarder sur les liens humains qui peinent à être tissés dans un contexte de forte individualisation. "Le développement des maisons en rang d'oignons, c'est une catastrophe au niveau sociologique, économique, paysager... » 
Figure 1. Trois schèmes de pensée d'approche des paysages

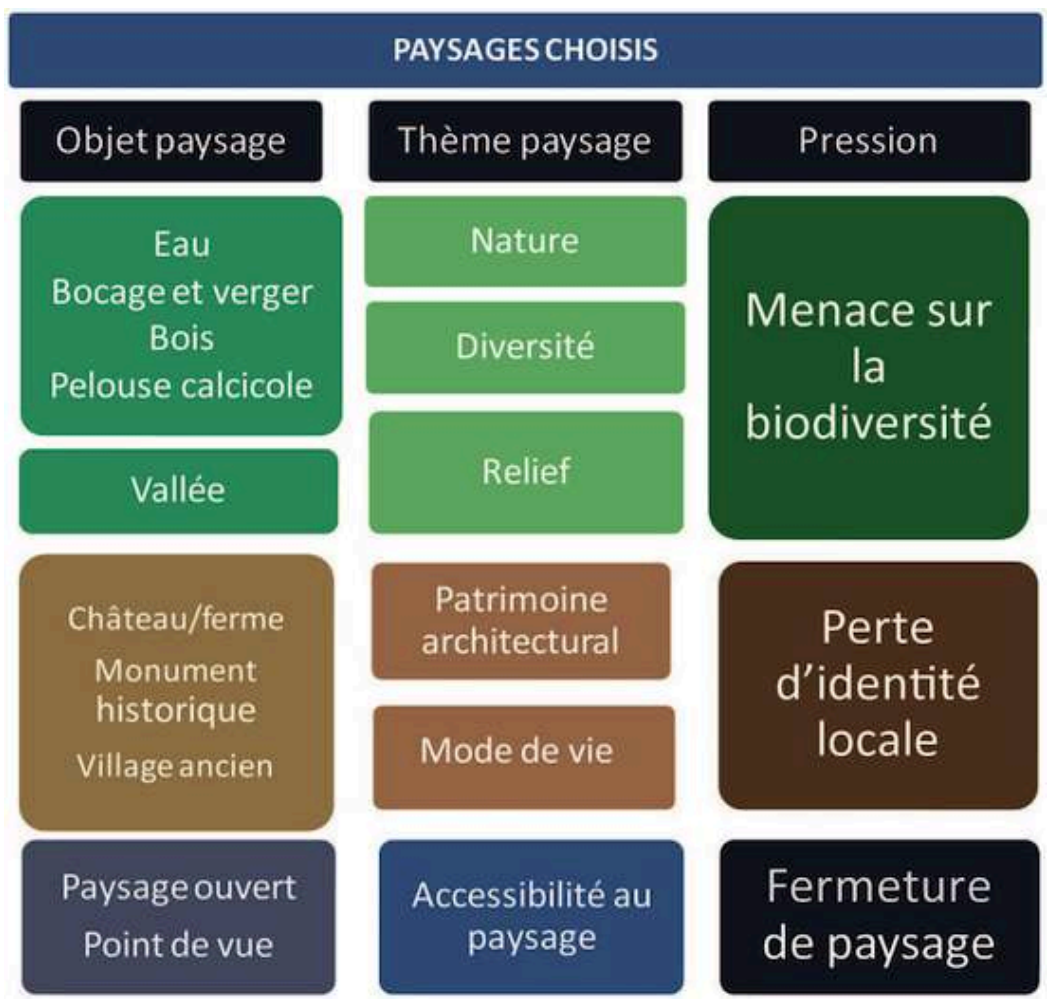

Lorsque les acteurs se plongent dans leurs paysages préférés, la composition qui se met spontanément en place, au fur et à mesure de l'énonciation des lieux, fait ressortir avec force cinq qualités déterminantes (figure 2). C'est l'appel à l'histoire et à la culture par un référent patrimonial ; c'est aussi la sélection d'un site naturel, comme les pelouses calaminaires ou des tourbières enherbées et fleuries; ce sont les habitants et les liens sociaux que leurs modes d'habiter laissent supposer. Les discussions sont ainsi généralement ponctuées par une énonciation nostalgique de villages "préservés »; c'est enfin la recherche de relief et de diversité comme qualités incontournables. On peut donc considérer que ces cinq dimensions de la construction globale et systématique des paysages appréciés participent de la valeur identitaire des paysages et doivent être intégrées dans la gestion, l'aménagement et la protection des paysages. 
Figure 2. Les cinq qualités des paysages choisis

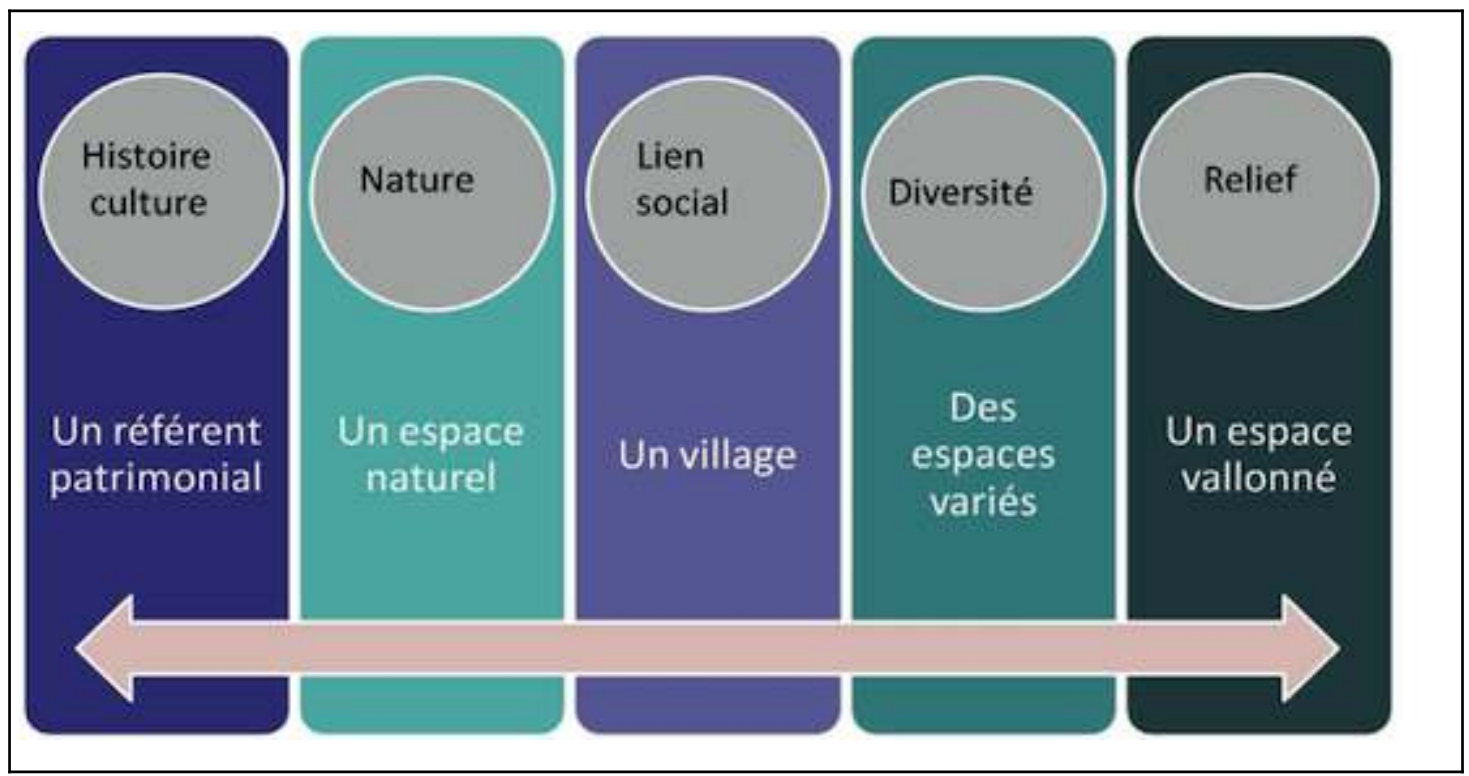

\section{Conclusion}

Bien que les publications soient encore récentes, selon les retours des acteurs de terrain, il est permis de penser que ces atlas des paysages servent de socle commun pour les acteurs susceptibles d'agir ou d'interagir sur le paysage. Les commissions communales consultatives d'aménagement du territoire et de mobilité ou les groupes d'actions locales se basent sur les atlas déjà parus pour justifier ou motiver leurs avis à propos de projets d'aménagement du territoire.

21 Les atlas sont aussi l'occasion de montrer que la construction d'une identité basée sur le paysage est aujourd'hui en voie d'élaboration mais qu'elle devrait peut-être s'ouvrir et intégrer les nouveaux facteurs d'évolution des paysages, pour éviter de tomber dans une forme de passéification. Le terreau pour une prise en compte collective des paysages est bel et bien présent. À chacun d'être conscient de l'intérêt de le laisser s'exprimer.

Les auteurs remercient les chercheurs qui ont participé à l'élaboration des différents atlas : pour ULB -l'IGEAT : Etienne Castiau, Nathalie Feremans, Stéphanie Queriat, Claire Neuray, Marc Nielsen, Thaïs Pons; pour le Lepur-ULg : Véronica Cremasco, Claire Van der Kaa; pour le FUSAGx (Gembloux) : Céline De Witte, Anne Doguet, Vincent Vanderheyden. 


\section{BIBLIOGRAPHIE}

Berque, A., « Paysage, milieu, histoire », Cinq propositions pour une théorie du paysage, Seyssel, Champ Vallon, France, 1994.

Convention de Florence, URL : http://conventions.coe.int/Treaty/fr/Treaties/Html/176.htm.

Feltz, C. (sous la dir. scientifique de), Les territoires paysagers de Wallonie. Étude et documents, MRW, Conférence permanente du développement territorial (CPDT), 2004.

Godart, M.-F. et Feltz, C. (sous la dir. scientifique de), Atlas des Paysages de Wallonie. La Haine et la Sambre, MRW, Conférence permanente du développement territorial (CPDT), 2012.

Godart, M.-F. et Feltz, C. (sous la dir. scientifique de), Atlas des Paysages de Wallonie. Le Plateau condrusien, MRW, Conférence permanente du développement territorial (CPDT), 2011.

Godart, M.-F. et Feltz, C. (sous la dir. scientifique de), Atlas des Paysages de Wallonie. Les Plateaux brabançon et hesbignons, MRW, Conférence permanente du développement territorial (CPDT), 2009.

Godart, M.-F. et Teller, J., (sous la dir. scientifique de), Atlas des paysages de Wallonie. L'Entre-Vesdreet-Meuse, MRW, Conférence permanente du développement territorial (CPDT), 2007.

Droz, Y et Mieville-Ott, V (sous la dir. de), La Polyphonie du paysage, Lausanne, Presses polytechniques et universitaires romandes, 2005.

Ministère de l'Écologie et du Développement, « Méthode pour les atlas des paysages. Enseignements méthodologiques de 10 ans de travaux », 2004.

Poullaouec-Gonidec, Domon, G et Paquette, S, Paysages en perspective, Montréal, Les Presses de l'université de Montréal, 2005.

\section{RÉSUMÉS}

En réponse à la Convention européenne des paysages, la Wallonie en Belgique a entamé des travaux de caractérisation des paysages à travers notamment l'élaboration d'une collection d'atlas qui devront à terme couvrir toute la région. Le présent article expose dans les grandes lignes la manière dont ces atlas sont élaborés et en quoi ils peuvent servir d'outil de sensibilisation. En plus de la méthodologie générale qui précise l'approche pluridisciplinaire, un focus est établi sur la perception des paysages par les acteurs locaux. Nous présentons les éléments paysagers auxquels les observateurs sont les plus attachés. Il apparaît ainsi qu'un paysage apprécié est choisi parce qu'il relève d'une composition d'attributs alliant autant des aspects physiques, émotionnels et que de lien social. Enfin, la confrontation de l'analyse objective des paysages à celle plus subjective portée par des acteurs clés montre que les atlas sont une voie vers un dialogue entre aménageurs et utilisateurs du paysage.

In response to the European convention on landscapes, the region of Wallonia in Belgium has started to characterize its landscapes by producing a collection of atlases that will cover the whole region in the end. This article explains in general how these atlases were elaborated. In addition to the general methodology, which provides a multidisciplinary approach, focus is set on the perception of landscapes by the local stakeholders. Besides its general multi-disciplinary approach, the uniqueness of the method used, is its focus on the perception of landscapes by the 
stakeholders involved. As a result, the study reveals that a landscape is appreciated not only by its physical appearance but by the combination of physical, emotional and social aspects. The confrontation between the objective landscape analysis and the subjective view of the stakeholders involved proves that these landscape atlases are a means for dialogue between landscape planners and its users.

\section{INDEX}

Mots-clés : paysages, atlas, perception, aménagement du territoire

Keywords : landscapes, atlas, perception, regional planning policy

\section{AUTEURS}

\section{THAÏS PONS}

Sociologue, Thaïs Pons est chercheure à Université libre de Bruxelles-IGEAT pour la Conférence permanente du développement territorial (CPDT).

tpons[at]ulb.ac[dot]be

\section{MARIE-FRANÇOISE GODART}

Docteur en sciences, elle est professeur à l'Université libre de Bruxelles - IGEAT.-

mfgodart[at]@ulb.ac[dot]be

\section{MIREILLE DECONINCK}

Docteur en sciences, elle est collaboratrice scientifique à l'Université libre de Bruxelles-IGEAT. mdeconinck[at]ulb.ac[dot]be 